\title{
Use of anti-D immunoglobulin in the treatment of threatened miscarriage in the accident and emergency department
}

\author{
L Weinberg
}

\begin{abstract}
Background-The UK guidelines for the use of anti-D immunoglobulin for rhesus prophylaxis have been revised. Anti-D immunoglobulin is no longer recommended for $R h D$ negative women after a threatened miscarriage less than 12 weeks gestation. These patients are at risk of rhesus immunisation, and there should be a policy for their treatment in the accident and emergency (A\&E) department.

Design-A retrospective study over a 17 month period was conducted looking at women less than 12 weeks gestation who presented to an $\mathrm{A} \& \mathrm{E}$ department with a threatened miscarriage.

Objectives-To determine how many of these patients presented with heavy or repeated bleeding, or abdominal pain, and whether the guidelines for the use of rhesus prophylaxis were followed.

Results-112 women fulfilled the criteria for inclusion. Nineteen patients were $R h \mathbf{D}$ negative. Eighty three patients $(74.1 \%)$ presented with either abdominal pain or heavy or recurrent bleeding. Rhesus status was recorded in the $A \& E$ notes in only 15 patients $(13.3 \%)$. Ninety seven patients $(86.6 \%)$ were discharged without rhesus status being checked. Fifteen Rh D negative patients were discharged without being offered anti-D immunoglobulin. Conclusion-Many women who present to the $A \& E$ department with a threatened miscarriage of less than 12 weeks gestation have heavy or recurrent bleeding or associated abdominal pain. These patients have an increased risk of fetomaternal haemorrhage and the consequent development of haemolytic disease of the newborn is possible. It should be mandatory for the $A \& E$ department to record rhesus status. In the context of $A \& E$ medicine, anti-D immunoglobulin should still be offered to all non-immune $R h D$ negative women presenting with a threatened miscarriage less than 12 weeks gestation. (Emerg Med f 2001;18:444-447)
\end{abstract}

Accident an Department, Royal Cornwall Hospital, Truro, UK

Correspondence to: Dr Weinberg, 67 Carrine Road, Newbridge, Truro, Cornwall TR1 3XB, UK (weinberg14@aol.com)

Accepted for publication 25 January 2001

The accident and emergency (A\&E) department plays an important part in the treatment of women who present with a threatened miscarriage. Vaginal bleeding occurs in one fifth of recognised pregnancies before the 20th week, and of these over half end in a miscarriage. ${ }^{1}$ A\&E departments provide a 24 hour service and are often the first port of call for many women who develop bleeding during pregnancy. It is essential that there is a policy for treating these patients especially $\mathrm{Rh} D$ negative women, who are at risk of rhesus immunisation resulting in haemolytic disease of the newborn (HDN). HDN is a haemolytic anaemia of fetal $\mathrm{Rh}$ positive red blood cells, characterised by varying degrees of anaemia, hyperbilirubinaemia, oedema, cardiac failure, hepatosplenomegaly and life threatening neurological sequelae of encephalopathy and convulsions, which occurs when there is incompatibility between fetal and maternal blood groups.

Anti-D immunoglobulin has been used for rhesus prophylaxis in the United Kingdom since 1969, reducing the number of deaths attributed to rhesus alloimmunisation from $46 / 100000$ in 1969 to $1.6 / 100000$ in $1990 .^{2}$ However, sensitisation still occurs in several patients, often resulting from failed or delayed administration of anti-D immunoglobulin after a potentially sensitising event. ${ }^{3-5}$

When Rh D immunoprophylaxis was initially introduced in the UK only postnatal treatment was recommended. In 1976 the department of health further advocated the use of anti-D immunoglobulin to cover all abortions, irrespective of gestational age. This was the current management policy until March 1999, when a joint working group of the British Transfusion Society and the Royal College of Obstetricians and Gynaecologists revised the existing guidelines for the use of anti-D immunoglobulin for rhesus prophylaxis. ${ }^{6}$ The updated revised guidelines list all potential sensitising events for which anti-D immunoglobulin must be given (see box).

The updated 1999 guidelines differ from the previous guidelines in that anti-D immunoglobulin is now no longer recommended for $\mathrm{Rh} \mathrm{D}$ negative women presenting with a threatened miscarriage with a viable fetus less than 12 weeks gestation. This is because most vaginal bleeding of less than 12 weeks gestation is mostly from maternal vessels in the decidua or cervix and not from fetal vessels in the chorionic villi, thereby minimising the risk of fetomaternal haemorrhage. ${ }^{7}$

Fetomaternal haemorrhage is more common if the choriodecidual space is breeched, which occurs after therapeutic or complete miscarriage. Approximately $4 \%$ of women who have a therapeutic miscarriage will have a transplacental haemorrhage of $>0.2 \mathrm{ml}$ of fetal red 


Sensitising events during pregnancy for
which anti-D immunoglobulin must be
given to Rh D negative women.
- Abortion
Therapeutic termination of pregnancy-
any gestation
$\quad$ Ectopic pregnancy
$\quad$ Spontaneous complete miscarriage
$>12$ weeks gestation
- Closed abdominal injury
- Antepartum haemorrhage
- Intrauterine death
- Stillbirth
- Invasive prenatal diagnostic procedures
- External cephalic version
- Delivery of an Rh D positive fetus
- Intrauterine procedures

cells, and of these patients $4 \%-5 \%$ will become sensitised. ${ }^{8}$ Choriodecidual space bleeding does occur after a threatened miscarriage, although the volume of fetal red cells is smaller $(<0.1 \mathrm{ml})$. However, is has been shown in the literature that sensitisation can occur in up to $3 \%$ of women exposed to a transplacental haemorrhage of $<0.1 \mathrm{ml},{ }^{9}$ therefore it can follow a threatened miscarriage of less than 12 weeks gestation. It seems feasible that such events are even more common when bleeding is heavy, painful or recurrent, although there is no evidence to support this.

The revised national guidelines, ${ }^{6}$ however state that it is "still prudent" to administer rhesus D prophylaxis when bleeding is heavy, repeated or if there is associated abdominal pain, particularly if these events occur near to 12 weeks gestation. If the gestation period is unknown, it should be confirmed by ultrasound. If bleeding continues intermittently after 12 weeks gestation, anti-D immunoglobulin should be given at six weekly intervals. The dose of anti-D immunoglobulin is 250 IU (50 $\mu \mathrm{g})$, which should be administered by deep intramuscular injection, preferably into the deltoid muscle for optimal absorption, as soon

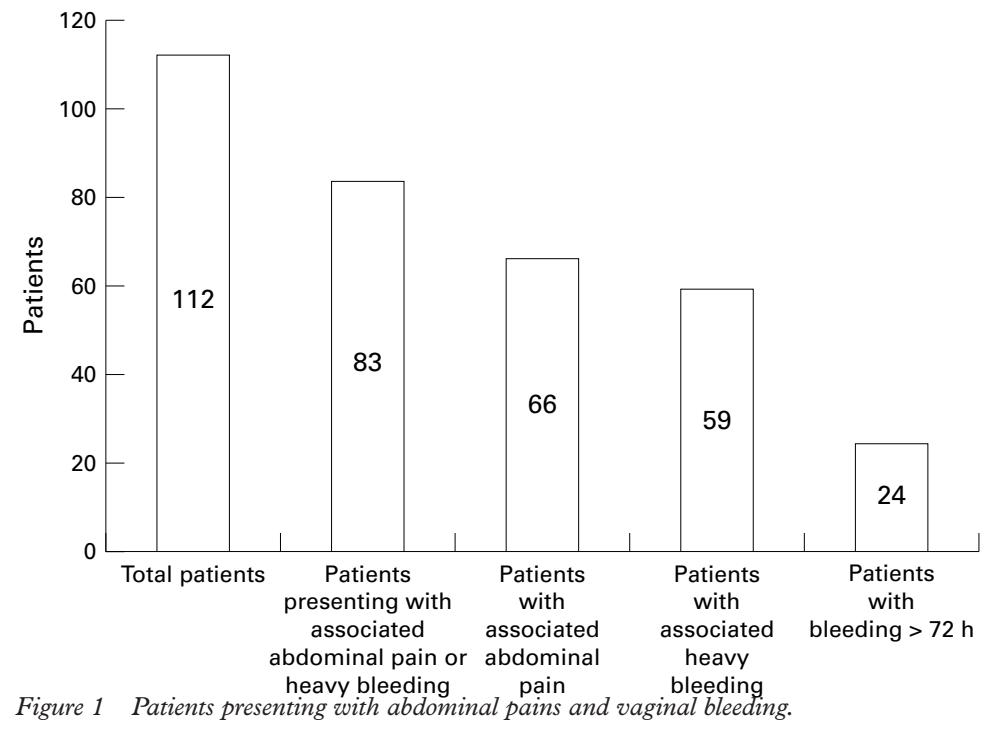

as possible after the sensitising event, but always within 72 hours. If anti-D immunoglobulin is not given within the first 72 hours, a dose given within 9-10 days may provide some protection.

The objectives of this study were to determine how many patients presenting to an $A \& E$ department with a threatened miscarriage of less than 12 weeks gestation had heavy or repeated bleeding, or associated abdominal pain, whether the guidelines, at the time of this study, for the use of anti-D immunoglobulin were followed, and to ascertain if the revised change to the 1999 UK guidelines concerning $\mathrm{Rh} \mathrm{D}$ prophylaxis now not being indicated for a threatened miscarriage of less than 12 weeks gestation is justified, in the treatment of such patients, in an A\&E department context.

\section{Methods}

The author conducted a retrospective study looking at pregnant women with a threatened miscarriage less than 12 weeks gestation who attended the A\&E department of the St James's University Hospital, Leeds, between 1 January 1998 and 31 May 1999 (17 months). The A\&E department assesses about 90000 patients each year and covers a population of 370000 .

Threatened miscarriage was defined as a patient who presented with pregnancy associated vaginal bleeding, and a positive immunological pregnancy test (urine or serum B HCG).

Gestational age was confirmed by the patient's antenatal records, which included the last normal menstrual period date, expected date of delivery or a dating ultrasound report. The gestational age in patients with no prior antenatal assessment, was calculated by the $\mathrm{A} \& \mathrm{E}$ doctor from the date of the patient's last normal menstrual period.

Only patients assessed and discharged solely by an A\&E doctor were included in the sample population. Patients referred to the on call gynaecologist by the $\mathrm{A} \& \mathrm{E}$ doctor for further assessment or management, and those patients admitted to the ward, were excluded.

Data collected included whether blood grouping for rhesus status was considered and recorded, whether anti-D immunoglobulin was administered to $\mathrm{Rh} \mathrm{D}$ negative women, and what follow up was arranged for the patient once discharged from the $\mathrm{A} \& \mathrm{E}$ department.

\section{Results}

Because of the nature of the study, there is no statistical validation for any of the figures. Patients' blood grouping results were obtained by the author at the end of the study period from the regional Blood Transfusion Service.

The results are summarised in figure 1 and figure 2. One hundred and twelve women fulfilled the criteria for inclusion of whom 19 were $\mathrm{Rh} \mathrm{D}$ negative. Rhesus status was recorded in the A\&E notes in only 15 patients (13.3\%), six of whom were $\mathrm{Rh} \mathrm{D}$ negative. Four of these $\mathrm{Rh} \mathrm{D}$ negative patients were given anti-D immunoglobulin in the $A \& E$ department. One hundred patients $(89.2 \%)$ 


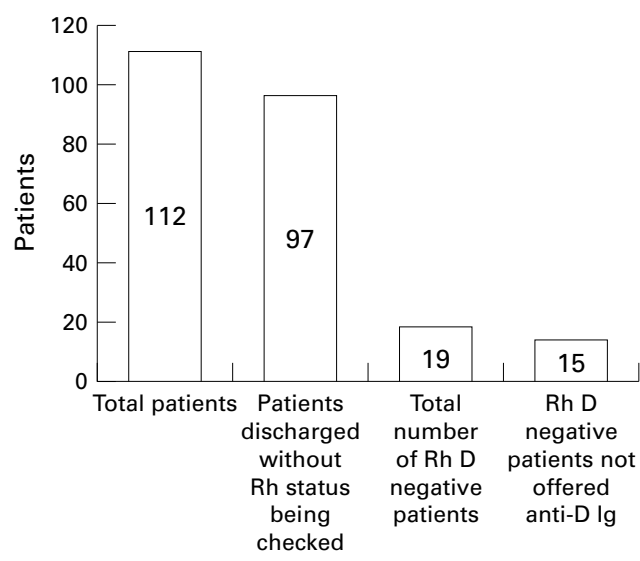

Figure 2 Rhesus D negative patients and administration of anti-D immunoglobulin.

were referred to an early pregnancy unit for further assessment and management.

\section{Discussion}

The study highlights the large proportion of women who present to the A\&E department with a threatened miscarriage of less than 12 weeks gestation, who have abdominal pain, or heavy or recurrent bleeding. It also emphasises the importance of checking and recording the patient's rhesus status.

It is clear that the guidelines for rhesus prophylaxis were not being followed. These results are comparable to research done in other A\&E departments. A prospective study including $109 \mathrm{~A} \& \mathrm{E}$ departments in England and Wales concluded that $77 \%$ of the departments did not administer anti-D when appropriate. ${ }^{10}$ Two other papers looking at the use of anti-D in the A\&E department confirmed rhesus status was not being recorded in $72 \%$ and $86 \%$ of their patients, and none of the rhesus $\mathrm{D}$ negative patients discharged, were offered anti-D immunoglobulin. ${ }^{11}{ }^{12}$

The crucial question that needs to be answered is why national guidelines are not being followed? The NHS Research and Development Directorate have already identified this dilemma, and there is no universal answer.

Many A\&E departments have facilities to refer pregnant women with a threatened miscarriage to an early pregnancy unit, and perhaps $A \& E$ doctors rely on these services for further assessment and continuation of treatment. This often is not optimal treatment for these patients, especially if they are rhesus D negative. Patients are frequently referred to the early pregnancy unit the following working day, and the recommended 72 hour cut off time for optimal benefit of anti-D immunoglobulin may be missed, especially if the patient has been bleeding for 72 hours at initial presentation (21.4\% of patients this study), or if they present on a Friday or bank holiday weekend, where they may only be seen by the assessment unit 24-72 hours later. A simple analogy would be someone with mild or atypical chest pain, being told to come back the following morning to the ECG clinic for an ECG to see if there are any ischaemic changes. This makes no sense, and the same principle applies to $\mathrm{Rh} \mathrm{D}$ negative women, who present with a threatened miscarriage. Anti-D immunoglobulin needs to be given immediately if indicated, avoiding the risk of sensitisation. Some patients may also default early pregnancy unit follow up.

Something needs to be done to optimise treatment of these patients. It would be ideal if we all knew our blood group and rhesus status or carried the information with us, but this is not practical. It is therefore mandatory for the A\&E department to check rhesus status at initial presentation, and if indicated, it should be the responsibility of the $\mathrm{A} \& \mathrm{E}$ doctor to offer anti-D immunoglobulin, minimising risk of rhesus sensitisation.

Human anti-D immunoglobulin is manufactured from high titre anti-D plasma, and has had an impeccable safety record for over 30 years. The manufacturing process is known to remove and/or inactivate HbsAg, HIV-1, HIV-2, and HCV. Side effects are minor, which may include pain at the site of the injection, rash and itching. There are medicolegal risks involved if sensitisation does occur without anti-D immunoglobulin being administered, and the consequences of sensitisation, which may include loss of pregnancy, loss of subsequent pregnancies and invasive procedures, outweigh the relatively insignificant risk involved of anti-D immunoglobulin being given, therefore even if there is uncertainty about gestational age, or the patient does not give a reliable menstrual history, anti-D immunoglobulin should still be prescribed.

Increased awareness for rhesus prophylaxis may be achieved by the introduction of a stamp or protocol form prompting the documentation of rhesus status, or by allowing nursing staff to check blood grouping and document the rhesus status result. This places additional pressure on an already overstretched nursing service, and the extent of this burden would need to be evaluated. New A\&E doctors need to be made aware that guidelines for the use of anti-D immunoglobulin are available, and updated guidelines should be available in all departments.

The revised updated guidelines for the use of anti-D immunoglobulin for rhesus prophylaxis exclude a threatened miscarriage of less than 12 weeks gestation as being a potentially sensitising event for which anti-D immunoglobulin should be given. There have been no randomised trials or cost-benefit analyses of anti-D immunoglobulin in threatened miscarriage, ${ }^{7}$ therefore the updated guidelines are no more evidence based than the previous guidelines that advocated its use for all miscarriages, including a threatened miscarriage of less than 12 weeks gestation.

This study clearly shows the high percentage of women who present to an A\&E department with a threatened miscarriage of less than 12 weeks gestation who have heavy or recurrent bleeding or associated abdominal pain, and that the guidelines, at the time of the study, for the use of anti-D immunoglobulin were not followed. These patients have an increased risk of fetomaternal haemorrhage and transplacental 
bleeding, and the consequent development of haemolytic disease of the newborn is possible.

It should be therefore be mandatory for all $A \& E$ doctors to document these patients's rhesus $\mathrm{D}$ status, and in the context of A\&E medicine, rhesus prophylaxis should still be offered to all non-immune $\mathrm{Rh} \mathrm{D}$ negative women who present with a threatened miscarriage, including those patients with a gestational age of less than 12 weeks.

I an grateful for the invaluable support and assistance of Dr Robin Illingworth, head of the A\&E Department at St James's University Hospital, Leeds, without whom this research would not have been possible, and to Dr Paul Gaffney, Research Registrar at St James's University Hospital for his advice and guidistrar at St James's University
ance in initiating this research.

Contributors

Dr Laurence Weinberg, designed the study, participated in data collection and documentation, formulated the primary objectives, analysed and interpreted the data, and wrote the paper. Funding: none.

Conflicts of interest: none.

1 Everett C. Incidence and outcome of bleeding before the 20 th week of pregnancy: prospective study of general prac20th week of pregnancy: p.
tice. BMF 1997;315:32-4.
2 Mollison PL, Eglefriet CP, Contreras M. Haemolytic disease of the fetus and newborn. In: Blood transfusion in clinical medicine. Oxford: Blackwell Science 1997, 543-91.

3 Howard HL, Martlew VJ, McFadyen IR, et al. A study of the incidence of, and reasons for $\mathrm{RhD}$ isoimmunisation in $\mathrm{RhD}$-negative pregnant women. [Abstract]. $\mathrm{Br} \mathcal{F}$ Obstet Gynaecol 1998;105:suppl 18.

4 Howard HL, Martlew VJ, McFadyen IR, et al. Preventing rhesus $\mathrm{D}$ haemolytic disease of the newborn by giving anti-D immunoglobulin: are the guidelines being adequately followed? Br f Obstet Gynaecol 1997;104:37-41.

5 McSweeny E, Kirkham J, Vinall P, et al. An audit of anti-D sensitisation in Yorkshire. Br f Obstet Gynaecol 1998;105: 1091-4.

6 Joint Working Group of the British Blood Transfusion Society and the Royal College of Obstetricians and Gynaecologist. Recommendations for the use of anti-D immu-
noglobulin for Rh prophylaxis. Transfus Med 1999;9:93-7.

7 Robson SC, Lee D, Urbaniak S. Anti-D immunoglobulin in RhD prophylaxis. Br f Obstet Gynaecol 1998;105:129-34.

8 Bowman JM. The prevention of Rh immunisation. Transfus Med Rev 1988;2:129-50.

9 Zipurski A, Israels LG, The pathogenesis and prevention of Rh immunisation. Can Med Assoc 7 1967;97:1254-7.

10 Gilling-Smith C, Toozs-Hobson P, Potts DJ, et al. Management of bleeding in early pregnancy in accident and emergency departments. BMF 1994;309:574-5.

11 Hyslop M, Grant J. Underutilisation of Rh prophylaxis in the emergency department: a retrospective survey. Ann Emerg Med 1991;21:181-3.

12 Huggon AM, Watson DP. Use of anti-D in an accident and emergency department. Arch Emerg Med 1993;10:306-9. 